\title{
THE TITRATION OF VACCINIA VIRUS BY THE POCK COUNTING TECHNIQUE
}

\author{
By C. KAPLAN \\ The Lister Institute of Preventive Medicine, Elstree, Hertfordshire \\ AND G. BELYAVIN \\ Department of Bacteriology, University College Hospital Medical School, \\ London, W.C. 1
}

(With 4 Figures in the Text)

Since the discovery that certain viruses produce discrete macroscopic lesions on the chorionic aspect of the chick allantoic membrane, this phenomenon has been widely used in virus titration. This method, together with bacteriophage plaque counting and plant virus titration by necrotic lesion counts on leaves, has been regarded as analogous to bacterial colony counting and involves the same two basic assumptions: $(a)$ that the growth medium is optimal for the microbe under consideration; and $(b)$ that each lesion, plaque or colony, is derived under optimal conditions from one infective particle. The information provided by such an assay is regarded as absolute, in that it gives a direct estimate of the number of infective units per unit volume, as opposed to the relatively imprecise indirect estimate of the number derived from a quantal titration. Counting techniques are reckoned on theoretical grounds to be superior to quantal assays not only for this reason but also because they are statistically subject to a lower error.

There is, however, little published work on the error of virus lesion counting techniques, although they have been extensively used, especially in experimental work with bacteriophages and now with animal viruses titrated in tissue cell monolayers.

Miles \& Misra (1938) and Davis (1940) showed that bacterial colonies from single drop inocula followed a Poissonian distribution, and it has been assumed that plaque and pock counts are distributed the same way.

No particular merit attaches to the fact that plaque, pock and colony counts conform to any one type of statistical distribution. The close approximation of such counts to a Poisson distribution would, however, carry the utilitarian advantage that the standard deviation of the mean count is then easily predictable, being equal to its square root.

A large series of vaccinia pock counts was carried out by one of us (C.K.) under routine conditions and, together with a similar series obtained from another laboratory, examined with the specific purpose of ascertaining whether (1) vaccinia pock counts follow a Poissonian distribution ; (2) if the distribution is not Poissonian, the class of distribution can be identified; and (3) any estimate can be obtained from these data of the standard deviation of vaccinia pock-count tritrations. The results of this analysis are presented here. 


\section{MATERIALS AND METHODS}

\section{Source of data}

Eighty-five chorio-allantoic membrane titrations of different preparations of vaccinia virus were performed over a period of several months, and the results of a further thirty titrations were kindly made available to us by Dr E. Boulter of the Microbiological Research Establishment, Porton. Most of these titrations were carried out using four to six eggs per dilution, and from these a total of ninetyseven groups of five membranes were selected, and the mean pock counts and variances calculated. The groups were arbitrarily selected, the only criteria being that each one represented a single homogeneous group, inoculated with the same dilution of virus within a given titration, that each membrane carried a countable number of pocks, and that a reasonable range of mean pock values was represented.

\section{Virus preparations used}

All preparations were derived from the Lister Institute vaccine strain of virus. Five types of preparation were used:

(1) Glycerolated small pox vaccine.

(2) Partially purified suspensions of virus prepared from sheep dermal pulp by one cycle of differential centrifugation.

(3) Reconstituted dried vaccine made by freeze-drying partially purified virus in $5 \%$ peptone (Collier, 1955).

(4) Suspensions of rabbit adapted virus of the 77th dermal passage partially purified by two or three cycles of differential centrifugation.

(5) Suspensions of chick embryo adapted virus prepared from infected chorioallantoic membranes. Some of these preparations were extracts clarified by horizontal centrifugation at $1000 \mathrm{~g}$ for $10 \mathrm{~min}$., while others were partially purified by two cycles of differential centrifugation. During the course of this work four successive single pock passages were made with this line of virus. This is analogous to the limiting dilution method of obtaining homogenous virus populations extensively used by Burnet and his school.

\section{Titration technique}

All dilutions were made in McIlvaine's sodium phosphate citric acid buffer, pH 7.2, 0.004 $\mathrm{m}$ phosphate. An initial 1/100 dilution of the material was made by delivering $0.1 \mathrm{ml}$. from a carefully calibrated Pasteur pipette into $9.9 \mathrm{ml}$. of buffer, with subsequent transfer of 1-9 ml. of diluent in 10-fold dilution series, and of 1-2.16 ml. of diluent in $\sqrt{ } 10$-fold series. When replicate titrations were made of any preparation, each set of dilutions was derived from its own 1/100 initial dilution.

At each dilution level tested, five 12-day old chick embryos were each inoculated with $0.1 \mathrm{ml}$. on the dropped chorio-allantoic membranes.

Chick embryos (Light Sussex-Rhode Island Red cross) were incubated at $38^{\circ} \mathrm{C}$. for 12 days with frequent automatic turning. After candling to determine the position of major blood vessels the shells were marked and drilled and the chorioallantoic membranes dropped by the method described by Beveridge \& Burnet 
(1946) as the 'standard technique'. The inoculum was introduced (generally within an hour) with a graduated Pasteur pipette, and its distribution over the dropped area of membrane facilitated by 'rocking' the egg to give it a fore-and-aft as well as side-to-side motion. After this the eggs were sealed with adhesive cellulose tape and incubated at $36^{\circ} \mathrm{C}$. for 2 days.

For counting the lesions the membranes were collected by cutting through the shell subequatorially in the long axis of the egg; the embryo, yolk sac and albumen sac were discarded and the infected area of chorio-allantoic membrane was left attached to the upper part of the shell. After trimming to a convenient size in situ, the lesion-bearing area was stripped from the shell with forceps, washed in $5 \%$ formalin and prepared for counting by immersion in water under a coverglass against a dark background.

After 2 days' incubation the pocks produced by all the sublines of the Lister Institute strain of vaccinia virus are dense white, clearly demarcated lesions about $1 \mathrm{~mm}$. diameter. Some have the central, depressed necrotic area considered to be typical of vaccinia, but this is generally not well developed until the third day of incubation. At 2 days secondary pocks are rare, but they are fairly numerous on the third day.

In the titrations analysed in this paper, membranes were accepted for counting if the pocks were discrete. Apart from those with confluent lesions, membranes were discarded only if they were grossly haemorrhagic or had such large nonspecific lesions, of the type described by Overman \& Tamm (1956), that specific lesions were quite uncountable. The incidence of such non-specific lesions was low in the groups of membranes considered here. No records have been kept until recently, but in 465 consecutive embryos lately inoculated only 23 non-specific lesions were noted; an incidence of $4.9 \%$. The lesions were distributed randomly throughout the membranes examined.

\section{Estimation of the standard deviation of the test}

However the result of a vaccinia pock titration is expressed arithmetically, the variability of this quantity stems from the variability of the actual pock counts themselves. It is reasonable to assume that the variability of the individual pock counts within any group reflects the general variability of pock counts carried out under the same conditions. The estimation of the error of the method as a whole, therefore, should rest on the accumulation of an adequate number of groups of pock counts and the computation of the group variances. From these, the overall variability of pock counts could be derived.

Theoretically such samples cannot of themselves yield a valid direct numerical estimate of the overall standard deviation, unless the nature of the statistical distribution of individual counts is first ascertained. In practice, however, it is only necessary to know how the variance, or standard deviation, is related to the sample mean and a graphical solution of the problem may be derived by plotting standard deviation against mean for a large series of samples. The resulting points will approximate to some characteristic function: parabolic for a Poissonian distribution; linear for data with a constant coefficient of variation. An approach. 
of this nature is not sufficiently exact to be used as a rigorous test of the nature of the distribution involved. Gross inconsistency with theoretical behaviour, however, would be apparent in a large series of samples, and as a considerable number of pock counts was available, this technique was used in the first instance.

\section{RESULTS}

The Poisson distribution having been so frequently invoked in the past to describe data of this nature, this characteristic was tested for first.

The variances of the ninety-seven five-membrane samples selected were plotted against the corresponding mean pock counts (Fig. 1). The relationship of variance to mean in each group of membranes deviates very markedly from that expected for values following a Poissonian distribution. It is similarly evident that variance

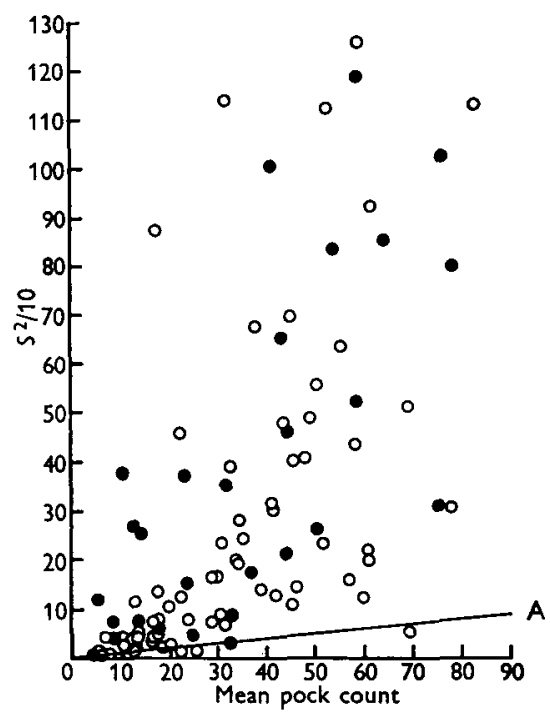

Fig. 1

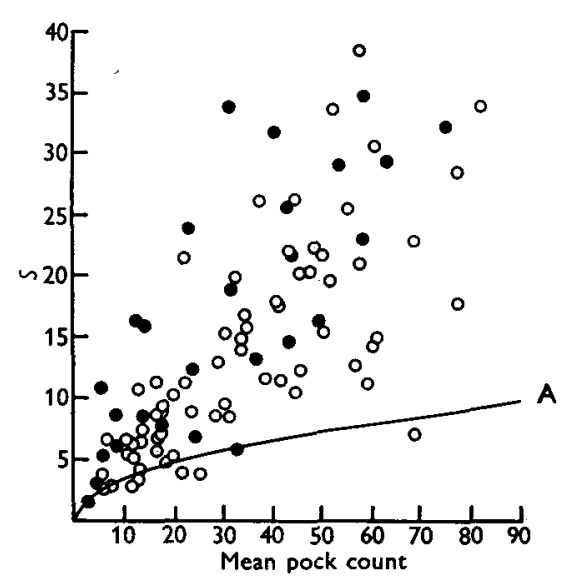

Fig. 2

Fig. 1. Variance $\left(S^{2}\right)$ against mean pock count. Variance plotted as $S^{2} / 10$ to compress the scale. $\mathbf{A}=$ expected line if distribution were Poissonian. $O$, Elstree titrations; 0 , Porton titrations.

Fig. 2. Standard deviation $(S)$ against mean pock count. A, expected line if distribution were Poissonian; O, titrations by CK (Elstree titrations); e, titrations by E. Boulter (Porton titrations).

and mean are not independent as would be the case if pock counts were 'normally' distributed. If the variances are replotted as standard deviations $(S)$ the resulting points do not approximate to any obvious linear function from which a coefficient of variation could be derived (Fig. 2). In fact, the individual values of $S$ lie scattered within a remarkably wide range, so that for a given mean pock count, the corresponding standard deviations may show as much as a fivefold or greater difference.

As the points represent values derived from groups of membranes inoculated with the same dilution of virus, irrespective of the titration from which they are derived, it may be argued that the coefficient of variation $(S / m)$ may be reasonably 
constant for a given titration. The observed scatter would then simply be a reflexion of the fact that $S / m$ varies from titration to titration according to the preparation being titrated, or the operator performing the assay.

To test this, a number of titrations giving countable pocks at several dilutions were selected from the series examined, and the mean pock count and standard deviation computed for each group of membranes inoculated at each dilution. The ratio $S / m$ was then plotted on a single ordinate for each titration (Fig. 3). This quantity varies widely within each titration, and extreme values of $S$ may be found at any dilution.

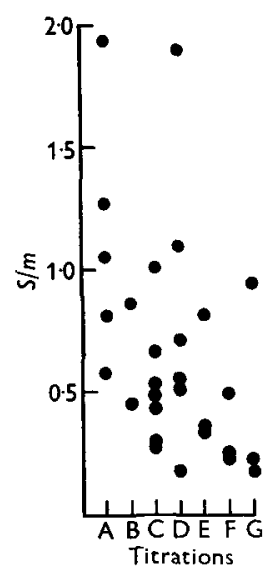

Fig. 3. Coefficients $(S / m)$ of variation within titrations.

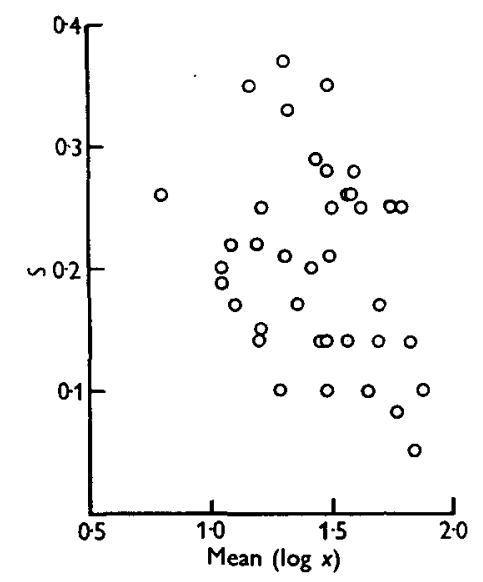

Fig. 4. Logarithmic transformation: standard deviation $(S)$ against mean $\log _{10}$ pock count.

\section{Log transformation}

It is well known that many distributions may be 'normalized' by a 'log transformation'.

The individual pock counts derived from forty-seven of the membrane groups studied were converted to $\log _{10}$ values. Means and standard deviations were again calculated and plotted graphically (Fig. 4).

The scatter of the individual points does not suggest a 'normal' distribution for the values.

\section{Reproducibility of titrations}

Several titrations were replicated in order to assess the reproducibility of mean pock counts. Two such groups of titrations are presented in Table 1. It is clear that excessively high or low mean pock counts may occur in the midst of reasonably concordant results. According to circumstances these could be interpreted as the result of experimental manipulation or of technical deficiency. The column of fiducial limits reveals, however, that all the means fall within the expected limits of variation and are a reflexion of the high variability of the individual membrane counts. In spite of the apparent reproducibility of mean pock counts, therefore, the scatter of individual pock counts precludes the certain detection of any but very large changes in virus concentration. 
Table 1. Two sets of replicate titrations of partially purified vaccinia virus preparations

\begin{tabular}{|c|c|c|c|c|c|}
\hline $\begin{array}{c}\text { Vaccinia } \\
\text { preparation }\end{array}$ & Titration & $\begin{array}{l}\text { Mean } \\
\text { pock } \\
\text { count }\end{array}$ & $\begin{array}{l}\text { Standard } \\
\text { deviation }\end{array}$ & $\begin{array}{c}\text { Standard } \\
\text { error of } \\
\text { mean }\end{array}$ & $\begin{array}{l}\text { Fiducial limits } \\
\qquad(p=0 \cdot 05)\end{array}$ \\
\hline \multirow[t]{4}{*}{$\mathbf{A}$} & 1 & $35 \cdot 7$ & $\pm 17 \cdot 5$ & $+7 \cdot 3$ & $16 \cdot 2-53 \cdot 8$ \\
\hline & 2 & $30 \cdot 1$ & \pm 21.9 & \pm 7.3 & $12 \cdot 8-47 \cdot 7$ \\
\hline & 3 & $19 \cdot 5$ & \pm 18.5 & \pm 7.7 & $\begin{array}{ll}0 & -39 \cdot 3\end{array}$ \\
\hline & 4 & $32 \cdot 4$ & \pm 15.5 & \pm 5.5 & $19 \cdot 4-45 \cdot 4$ \\
\hline \multirow[t]{4}{*}{ B } & 1 & $47 \cdot 8$ & $\pm 29 \cdot 78$ & $\pm 12 \cdot 1$ & $16 \cdot 6-79 \cdot 0$ \\
\hline & 2 & $41 \cdot 5$ & \pm 8.97 & \pm 3.7 & $32 \cdot 1-50 \cdot 9$ \\
\hline & 3 & $65 \cdot 0$ & $\pm 19 \cdot 3$ & \pm 8.6 & $41 \cdot 0-89 \cdot 0$ \\
\hline & 4 & $36 \cdot 3$ & $\pm 14 \cdot 25$ & \pm 5.8 & $21 \cdot 2-51 \cdot 4$ \\
\hline
\end{tabular}

\section{DISCUSSION}

Rigourous formal tests have not been applied to the data presented here, but it is, nevertheless, obvious that the pock counts do not conform to a Poisson distribution. Other workers have observed greater or lesser degrees of deviation of pock counts from this theoretical distribution (Kapsenberg, 1954; Fenner \& McIntyre, 1956; Rubin, 1955), and have offered hypotheses to explain these discrepancies.

Nearly all have assumed that the virus particles in the suspension used for inoculation will be distributed among samples in a Poissonian manner. That this is not reflected in the resulting pock counts is presumed to be due to heterogeneity of the egg membrane surface with respect to virus susceptibility. Such a hypothesis has been examined theoretically in some detail by Moran and Fazekas de St Groth (Moran, 1955; Fazekas de St Groth \& Moran, 1955) in connexion with the deviation of quantal egg infectivity titrations from the expected simple exponential form. These authors suggest that the Poissonian distribution generated by the sampling of suspended virus particles has superimposed upon it a secondary distribution of host susceptibility.

Again, Kleczkowski (1955) has shown that in the case of leaf plaque counts with tobacco necrosis virus, the count standard error approaches constancy as the mean count rises, and the counts themselves may therefore be supposed to tend to normality. It is possible that the departure of our counts from Poissonianism and the lack of stability in their variance could be explained as the result of the superimposition of two distributions.

Whatever the distribution of these pock counts is it is clearly desirable from purely practical considerations that the variance of the individual counts should be reduced and stabilized. When occasional large deviations of mean count occur the results of experimental manipulation cannot always be distinguished with certainty from chance variation without the use of statistical tests which are the only objective means of selection available. It would be useful, therefore, to concentrate on the reduction and stabilization of the pock count variance as formal statistical tests are of doubtful validity until the 'wandering' variance of individual pock counts is controlled. Westwood, Phipps \& Boulter (1957) claim that this may 
be achieved if details of membrane handling technique are rigorously adhered to.* Overman \& Tamm (1956) have shown that the development of non-specific lesions on the chorio-allantoic membrane greatly reduces the mean pock count obtained at a given dilution of virus. If precautions are taken against non-specific lesions the mean pock counts in replicate titrations increase, but the count variances are by no means stabilized as can be seen if the results of individual membrane counts tabulated by these authors are analysed by standard statistical techniques.

It has also been shown (Kapsenberg, 1954) that the physical state of the virus suspension with regard to both purity and dispersion does not affect the variance of the observed mean pock counts, and this has been confirmed by us. The pock count variances of crude glycerolated lymph preparations were as scattered as those of more purified suspensions.

An empirical approach to the precision of pock count titrations was adopted by Overman \& Tamm (1956) who assumed that if the mean pock counts at successive dilutions bore the expected relationship to each other, then the titration was internally consistent and the result one of quite considerable precision. This manoeuvre, however, only tests the linearity of the response within the titration, and as the slope of the response line does not affect the variance of the mean pock count at any dilution it cannot exclusively determine the precision of the test. For some purposes, such as the determination of vaccine potency, great precision of testing is not really necessary. In titrations of this sort the recognized inadequacies of the method may well be outweighed by the advantages of convenience and sensitivity.

It is possible that improvement in technique may stabilize the pock count variance without reducing it appreciably. Nevertheless, this would at least permit a valid estimate of the overall variance of the test and hence of its intrinsic reproducibility.

\section{SUMMARY}

Ninety-seven groups of five-membrane pock counts from 115 titrations of vaccinia virus preparations were examined in an attempt to identify the statistical distribution followed by such counts, and to obtain an estimate of the overall coefficient of variation of the technique.

It was found that the distribution of counts was neither Poissonian nor normal, and that the count variances 'wandered' so extensively that no valid estimate of the overall coefficient of variation was possible. Nor was it possible to assess the intrinsic reproducibility of the test, although it is empirically a useful one.

\section{REFERENCES}

Beveridge, W. I. B. \& Burnet, F. M. (1946). The cultivation of viruses and rickettsiae in the chick embryo. Spec. Rep. Ser. med. Res. Coun. Lond., no. 256.

Collier, L. H. (1955). The development of a stable smallpox vaccine. J. Hyg., Camb., 53, 76. Davis, H. (1940). A quantitative bacteriological investigation of the Tyndallisation process. Quart. J. Pharm. 13, 14.

* The results given us by Dr Boulter were obtained by the standard technique described here. 
Fazekas de st Groth, S. \& Moran, P. A. P. (1955). A mathematical model of virus-cell interaction. J. Hyg., Camb., 53, 291.

Fenner, F. \& McIntyre, G. A. (1956). Infectivity titrations of myxoma virus in the rabbit and the developing chick embryo. J. Hyg., Camb., 54, 246.

KAPSEnBerg, J. G. (1954). Het Kweken van Vaccinia-virus in geexplanteerd Weefsel. Amsterdam: (M.D. Thesis).

KIEczkowski, A. (1955). The statistical analysis of plant virus assays: a transformation to include lesion numbers with small means. J. gen. Microbiol. 13, 91 .

Miles, A. A. \& Misra, S. S. (1938). The estimation of the bactericidal power of the blood. J. Hyg., Camb., 38, 732 .

Moran, P. A. P. (1955). Measuring resistance to virus infection. J. Hyg., Camb., 53, 143.

Overman, J. R. \& TAMm, I. (1956). Quantitative titration of vaccinia virus on the chorioallantoic membrane. J. Immunol. 76, 228.

RuBin, H. (1955). Quantitative relations between causative virus and cell in the Rous No. 1 chicken sarcoma. Virology, 1, 445.

Westwood, J. C. N., Phipps, P. H. \& Boulter, E. A. (1957). The titration of vaccinia virus on the chorio-allantoic membrane of the developing chick! embryo. J.Hyg., Camb., $55,123$.

(MS. received for publication 6. II. 57) 